\title{
Analysis of loop voltage evolution in current drive experiments in the Phaedrus-T tokamak
}

\author{
C. Litwin, N. Hershkowitz, S. Wukitch, T. Intrator, M. Vukovic, D. Brouchous, R. Breun, \\ and M. Harper \\ Department of Nuclear Engineering and Engineering Physics, University of Wisconsin-Madison, Madison, \\ Wisconsin 53706-1687
}

(Received 3 April 1995; accepted 1 September 1995)

The loop voltage response in the low-frequency current drive experiments is analyzed in order to extract information about the current drive profile and efficiency. (c) 1995 American Institute of Physics.

\section{INTRODUCTION}

Recent experiments in the Phaedrus-T tokamak ${ }^{1}$ showed large loop voltage drops when a pair of antennas with $\pi / 2$ relative phasing were turned on at a frequency below the ion cyclotron frequency. These results have been interpreted as the evidence for current driven by propagating Alfvén waves. The loop voltage measured near the plasma edge exhibited a somewhat unexpected behavior: the initial loop voltage drop occurred on a time scale much shorter than the $L / R$ time inferred from current decay experiments and was followed by a much slower increase. The purpose of the present paper is to provide a quantitative analysis of the loop voltage response in the above described experiments.

\section{DISCUSSION}

Experiments described in Ref. 1 were conducted in plasma with peak density and electron temperature equal to $3 \times 10 \mathrm{~cm}^{-3}$ and $500 \pm 100 \mathrm{eV}$, respectively, toroidal current $65 \mathrm{kA}$, and toroidal magnetic field on the axis $6.8 \mathrm{kG}$. The radial density and temperature profiles were approximately parabolic and square parabolic, respectively. During the current drive experiments, the total plasma current has been held constant, in order to minimize the inductive effects. The observed loop voltage drop was associated with little temperature or density change, despite the fact that the emitted radio frequency $(\mathrm{RF})$ power exceeds the Ohmic power by the factor of 3 . The reason for this constancy of stored thermal energy is not entirely understood. Two main possibilities are: the energy confinement may be degraded by the application of RF; or only a small fraction of the emitted RF power may be absorbed in the bulk of the plasma. Upon the RF turn-on a rise in the bremsstrahlung emission has been observed, ${ }^{2}$ indicating an increase in the effective ion charge $Z_{\text {eff }}$. The fast $Z_{\text {eff }}$ rise time $(\sim 2 \mathrm{~ms})$ is consistent with the confinement degradation by RF.

The loop voltage and RF power variation in one of typical and reproducible discharges described in Ref. 1 are shown in Figs. 1 and 2. The RF power reaches two-thirds of its peak value during the first $0.5 \mathrm{~ms}$, then rises the remaining one-third over $10 \mathrm{~ms}$, subsequently decaying slowly over a longer time scale. In Fig. 3 the time evolution of the bremsstrahlung emission from the central cord is shown, exhibiting a rapid (on the time scale of $2 \mathrm{~ms}$ ) rise following the $\mathrm{RF}$ turn-on. The $Z_{\text {eff }}$ profiles, measured $2 \mathrm{~ms}$ before and $2 \mathrm{~ms}$ after the RF turn-on, are flat within experimental uncertainty in the bulk of the plasma (Fig. 4). The average $Z_{\text {eff }}=1.8 \pm 0.5$ in the Ohmic phase is consistent with the measured plasma resistance of $13.5 \mu \Omega$ that implies $Z_{\text {eff }}^{\prime}=1.9$ for the temperature profile obtained from the Thomson scattering. ${ }^{1}$ Upon the $R F$ turn-on, $Z_{\text {eff }}$ rises to $2.5 \pm 0.5$.

The $Z_{\text {eff }}$ profile flatness, together with the constancy of the electron temperature profile, allows us to represent the corresponding Spitzer conductivity as a separable function of space and time: $\sigma(r, t)=\zeta(t) \hat{\sigma}(r)$, with $\hat{\sigma}$ normalized in such a way that $\zeta=1$ in the Ohmic phase. The toroidal electric field diffusion in such a case has been analyzed in the companion paper. ${ }^{3}$ Characterizing current drive by the source function $S=J_{\mathrm{cd}} / \hat{\sigma}$, where $J_{\mathrm{cd}}$ is the driven current density, the edge loop voltage change, for a constant total plasma current, can be expressed as

$$
\Delta \tilde{V}(\tau) \equiv \tilde{V}(\tau)-V_{0}=-\bar{V}_{S}(\tau)(1+\delta),
$$

with

$$
\begin{aligned}
\delta(\tau)= & \frac{1}{\bar{S}(\tau)} \sum_{n=1}^{\infty} F_{n}(a) e^{-\lambda_{n} \tau}\left(S^{(n)}\left(0^{+}\right)\right. \\
& \left.+\int_{0}^{\tau} d \tau^{\prime} e^{\lambda_{n} \tau^{\prime}} \frac{d S^{(n)}}{d \tau^{\prime}}\right)
\end{aligned}
$$

Here $\quad \tilde{V}(\tau)=2 \pi R_{0} \zeta(\tau) E(a, \tau), \quad V_{0}=V(0), \quad \bar{V}_{S}(\tau)$ $=2 \pi R_{0} \bar{S}(\tau)$, with $R_{0}$ and $a$ being the major and minor radius, respectively; $E(r, \tau)$ is the toroidal electric field and $\bar{S}=\left\langle J_{\mathrm{cd}}\right\rangle\langle\hat{\sigma}\rangle$, with \langle\rangle denoting the volume average; $\lambda_{n}$ and $F_{n}$ are eigenvalues and eigenfunctions corresponding to the Laplace-transformed diffusion equation; $S^{(n)}$ is the projection of the source function on the eigenfunction $F_{n} ; \tau$ $\left.\equiv \int_{t_{0}}^{t} d t^{\prime} \gamma_{R}\left(t^{\prime}\right)\right]$, with $t_{0}$ and $\gamma_{R}=c^{2} / 4 \pi \sigma(0, t) a^{2}$ being the beginning of the RF pulse and the central resistive diffusion rate, respectively. For a separable source function, in the weak time-dependence limit,

$$
\delta(\tau)=\sum_{n=1}^{\infty} F_{n}(a)\left(\frac{\bar{S}(0)}{\bar{S}(\tau)} e^{-\lambda_{n} \tau}+\frac{1-e^{-\lambda_{n} \tau}}{\lambda_{n} \tau_{S}}\right) \frac{S_{n}(\tau)}{\bar{S}(\tau)},
$$

where $\tau_{s}=1 /(d \ln \bar{S} / d \tau)$ is the current drive variation time scale. The term proportional to $S(0)$ is retained in Eqs. (2a) and $(2 b)$ in order to permit an instantaneous rise of the current drive at $t=0$. 


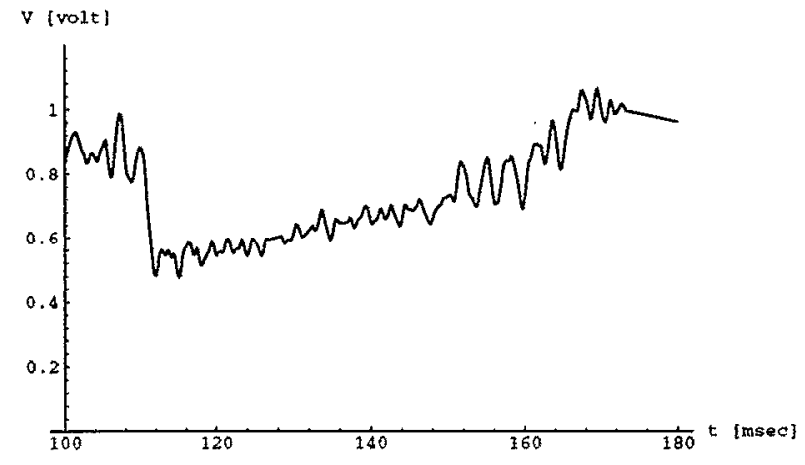

FIG. 1. Time dependence of the measured loop voltage.

To find eigenfunctions and eigenvalues, we assume, as suggested by Thomson scattering temperature measurements, ${ }^{1}$ that the conductivity profile has the form $\sigma(r)=\sigma_{0}\left(1-r^{2} / a^{2}\right)^{3}$. The eigenfunctions are found by numerically solving the eigenvalue equation

$$
\frac{1}{x} \frac{d}{d x}\left(x \frac{d F}{d x}\right)+\lambda\left(1-x^{2}\right)^{3} F=0
$$

$(x \equiv r / a)$ subject to the current constancy condition

$$
\left.\frac{d F}{d x}\right|_{x=1}=0 \text {. }
$$

The current drive by means of Alfvén waves is expected to be localized in the vicinity of the Alfvén wave resonance. This expectation is in part confirmed by the reflectometry measurements of density fluctuations during the RF pulse. ${ }^{4}$ These measurements showed that fluctuations were localized to regions with half-width $d \sim 0.1 a$ at $x \equiv r / a \sim 0.5$. Although they were performed at higher densities than the current drive experiments discussed here, we shall nonetheless use these results as a guide in choosing an appropriate form of the source function. We shall assume the source function in the form of a Gaussian: $S \sim \exp \left[-\left(x-x_{0}\right)^{2} / 2 d^{2}\right]$. For such a source only the lowest $n_{\max } \sim a / d$ modes contribute significantly to the sum in Eq. (2a).

Taking the above into account, we can now determine the current drive location by comparing the measured and the computed loop voltage responses for various values of $x_{0}$

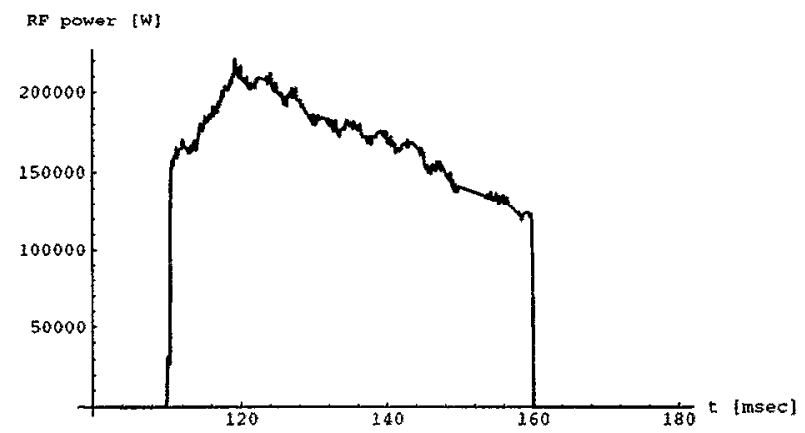

FIG. 2. Time dependence of the RF power.

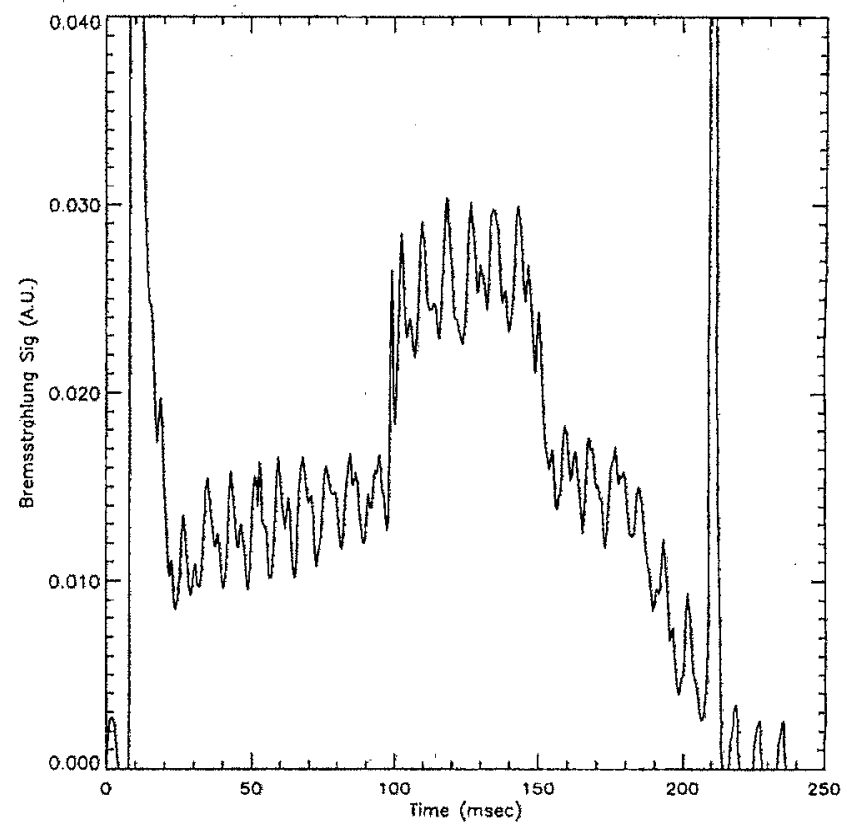

FIG. 3. Time dependence of $Z_{\text {eff }}$ from the central chord.

and a/d. Since the magnitude of the current drive is not known independently, we consider the relative loop voltage change,

$$
\mu \equiv \frac{\Delta \tilde{V}}{\Delta \tilde{V}_{1}} \frac{f_{1} P_{1}}{f P},
$$

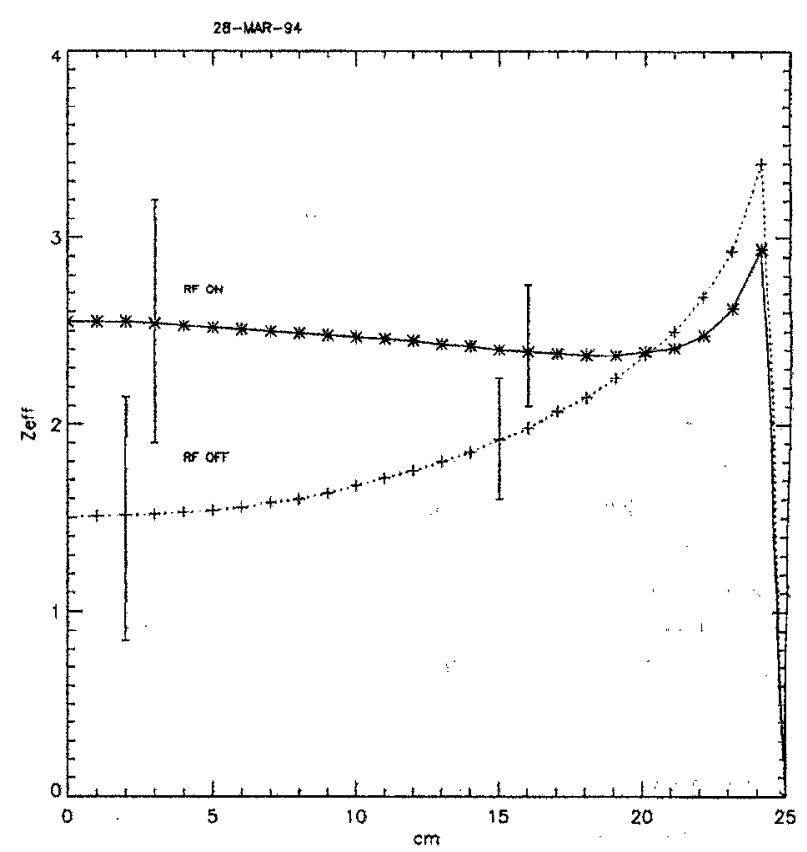

FIG. 4. Radial profile of $Z_{\text {eff }}$ before (dashed line) and after (solid line) the RF turn-on. 


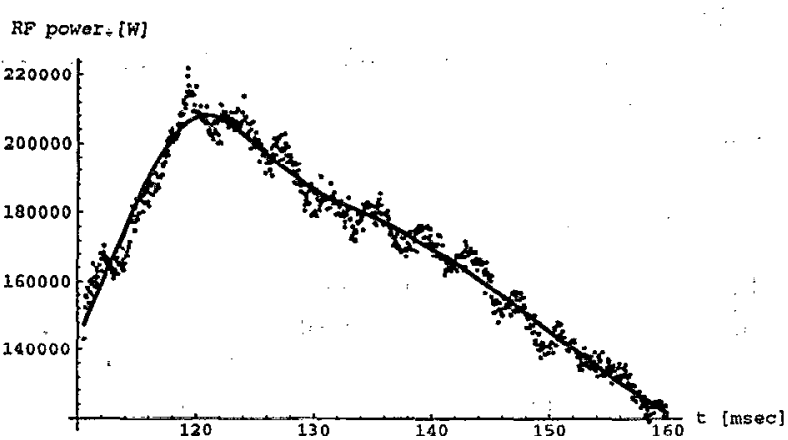

FIG. 5. Analytic fit (solid line) to the measured RF power (dots).

where $f$ is the current drive efficiency, $P$ is the RF power, and the subscript 1 refers to a certain arbitrarily chosen time $t_{1}$ during the RF pulse (we choose the middle of the RF pulse). From Eq. (1) it follows that

$$
\mu=\frac{1+\delta}{1+\delta_{1}} \text {. }
$$

In our analysis, we shall assume that the current drive efficiency is constant during the RF pulse so that $\bar{S}$ is proportional to the RF power. This assumption is presumably not valid during the first $2 \mathrm{~ms}$ of the pulse during which $Z_{\text {eff }}$ is evolving. Using the Ehst-Karney formula ${ }^{5}$ we estimate that the current drive efficiency is reduced by less than $20 \%$ as $Z_{\text {eff }}$ increases from 1.9 to 2.5 . Thus the loop voltage change during the first $2 \mathrm{~ms}$ may deviate by $20 \%$ from the correct value. We shall ignore this small error, noting that it is smaller than the experimental uncertainty in the plasma resistance.

We treat the initial power rise (during the first $0.5 \mathrm{~ms}$ after the RF turn-on) as instantaneous. The time scale of its subsequent variation ( $>25 \mathrm{~ms}$ ) is longer than the current relaxation time scale, which for experimental parameters is in the range of $10-15 \mathrm{~ms}$. In our calculation, the contribution of $n=1$ and $n=2$ terms in Eq. (2a) is calculated exactly, while $n>2$ terms are approximated by their weak timedependence limits [cf. Eq. (2b)].

We obtain $\tau_{S}$ from the RF power variation. To do that we first find a smooth analytic fit to the data, shown in Fig. 3;

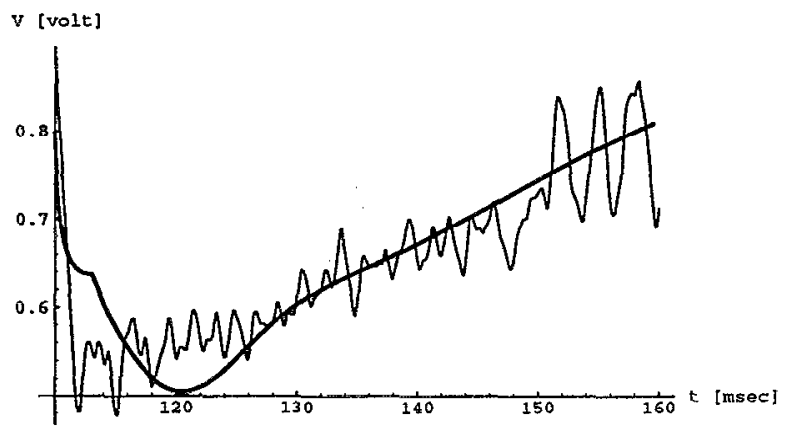

FIG. 6. Comparison of the measured (thin solid line) and calculated loop voltage for $\zeta_{\mathrm{RF}}=0.81$ and a Gaussian current drive profile with $x_{0}=0.50$ and $d / a=0.2$ (thick solid line) and $x_{0}=0.55$ and $d / a=0.2$.

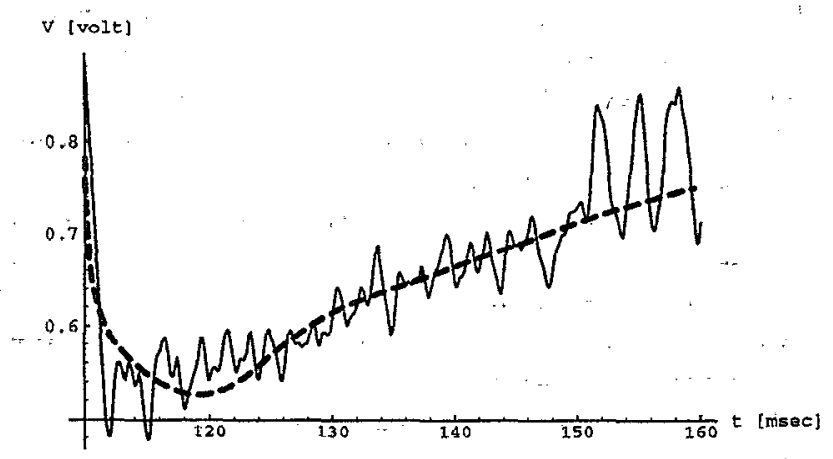

FIG. 7: Comparison of the measured (solid line) and calculated loop voltage for $\zeta_{\mathrm{RF}}=1$ and a Gaussian current drive profile with $x_{\mathrm{n}}=0.58$ and $d / a=0.18$ (dashed line).

and with its aid we calculate $\tau_{S}$. The time dependence of $\zeta$ is obtained with the aid of the Spitzer-Härm formula, ${ }^{6}$

$$
\zeta=\frac{T_{e}^{3 / 2}}{Z_{\text {eff }}}\left(0.12+\frac{0.192}{1.077+Z_{\text {eff }}}\right)^{-1},
$$

where $T_{e}$ is in $\mathrm{keV}$ and $\zeta$ is normalized so that $\zeta=1$ in the Ohmic phase, with $T_{e}=0.5 \mathrm{keV}$ and $Z_{\text {elf }}=1.9$. We use Eq. (7) to estimate the central resistive diffusion time during the RF pulse. In the Ohmic phase the plasma resistance is equal to 13.5 $\mu \Omega$, which for the assumed conductivity profile implies that $\gamma_{R}^{-1} \approx 690 \mathrm{~ms}$. Assuming, consistently with measurements, that $T_{e}=0.5 \mathrm{keV}$ and $Z_{\text {eff }}=2.5$ during the RF pulse, Eq. (7) implies that $\gamma_{R}^{-1} \approx 560 \mathrm{~ms}$.

We calculate the loop voltage response with the aid of Eqs. (5)-(7), from the RF power and plasma resistance variation for various values of $x_{0}$ and $d / a$. As suggested by the bremsstrahlung emission, the plasma resistance is assumed to rise linearly during the first $2 \mathrm{~ms}$ of the RF pulse and remain subsequently constant: $\zeta=\zeta_{\mathrm{RF}}=$ const. From Eq. (7) it follows that for measured values of $T_{e}$ and $Z_{\text {eff }}, \zeta_{\mathrm{RF}}=0.81 \pm 0.27$. The dependence of the loop voltage response on the width of the current drive region in the range of $d / a \sim 0.02-0.2$ is relatively weak, but the response is quite sensitive to the current drive location. For $\zeta_{R F}=0.81$ (corresponding, e.g., to fixed $T_{e}$ and $Z_{\text {eff }}$ increasing from 1.9 to 2.5) the best fit to the measured loop voltage response is shown in Fig. 5. The driven current profile in this case is characterized by $x_{0}=0.50$ and $d / a=0.2$. The calculated loop voltage response has a qualitatively similar shape to the measured one, although it deviates in detail in the interval between $t=112$ and $t=124 \mathrm{~ms}$. This might be due to a more complicated than assumed current drive profile (a more satisfactory fit is, in fact, found for a two-peak profile). Better fits can be obtained for higher values of $\zeta_{\mathrm{RF}}$. For example, for $\zeta_{\mathrm{RF}}=1$, which is within the experimental uncertainty from $\zeta_{\mathrm{RF}}=0.81$, a good fit is found for $x_{0}=0.58$ and $d / a=0.18$ (Fig. 6).

In the above analysis we have chosen a Gaussian profile for the current source. In reality, the current drive profile may be more complex, consisting of several peaks that correspond to Alfvén resonances of modes with different toroidal and poloidal numbers. If the loop voltage response and the conductivity magnitude and profile were known exactly, one 
could, in principle, reconstruct even such a complicated current drive shape. In the present case, however, the experimental uncertainties make it impossible to distinguish between a single broad peak and two (or more) relatively closely spaced peaks, as we saw above.

Assuming that the current is driven at a single Alfvén wave resonance, the knowledge of the current drive location can be exploited to determine the parallel refraction index of the wave. We employ the Alfvén wave resonance condition in the eikonal approximation ${ }^{7}$ for a hydrogen plasma with a $4 \%-5 \%$ carbon impurity (corresponding to $Z_{\text {eff }} \sim 2.2-2.5$ ). From it we deduce that a mode with toroidal and poloidal numbers $n$ and $m$ resonates at $x \sim 0.5-0.6$ if $n+m / q \sim 3.7-$ 4.3 ( $q \sim 1.3-1.5$ is the safety factor) for the parameters of the present experiment. This result agrees qualitatively with the modeling ${ }^{8}$ based on the toroidal cold plasma code LION. ${ }^{9}$ The latter indicates that the dominant absorption occurs for modes with $n=5$ and $m= \pm 1, \pm 2$; and that the mean radius of absorption for experimental parameters is located about $x \sim 0.5-0.6$.

The driven current magnitude is determined from

$$
\frac{\left\langle J_{\mathrm{cd}}(\tau)\right\rangle}{\langle J\rangle}=\frac{\Delta \tilde{V}(\tau)}{V_{0}[1+\delta(\tau)]} .
$$

In the middle of the pulse $(t=135 \mathrm{~ms})$ and for the above parameters, $\delta$ is negligible and $\Delta \tilde{V} / V_{0}=0.42$ for $\zeta_{\mathrm{RF}}=0.81$. This implies that $27 \mathrm{kA}$ of current are driven by the nominal $180 \mathrm{~kW}$ of RF power with the corresponding current drive efficiency of $0.15 \mathrm{~A} / \mathrm{W}$. This corresponds to $32 \mathrm{kA}$ of current driven at the peak power of $210 \mathrm{~kW}$. For $\zeta_{\mathrm{RF}}=1$, the deduced current drive efficiency is $0.1 \mathrm{~A} / \mathrm{W}$. The actual current drive efficiency is likely to be higher since not all the RF power reaches the plasma core.

\section{CONCLUSION}

In summary, we have analyzed the loop voltage evolution during Alfvén wave current drive experiments at the Phaedrus-T tokamak. We find that the observed loop voltage response is consistent with an off axis current drive. The current drive is found to occur at $r / a \approx 0.5$ with the efficiency $f>0.1-0.15 \mathrm{~A} / \mathrm{W}$.

\section{ACKNOWLEDGMENT}

This research has been supported by the U.S. Department of Energy Grant No. DE-FG02-88ER53264.

${ }^{1} \mathrm{~S}$. Wukitch, M. Vukovic, R. Breun, D. Brouchous, D. A. Diebold, M. Doczy, A. Elfimov, D. Edgell, N. Hershkowitz, T. Intrator, M. Kishinevsky, C. Litwin, P. Moroz, and P. Probert, Phys. Rev. Lett. 74, 2240 (1995).

${ }^{2}$ D. Brouchous, D. Edgell, and D. Diebold, Bull. Am. Phys. Soc. 39, 1631 (1994).

${ }^{3}$ C. Litwin, Phys. Plasmas 2, 4542 (1995).

${ }^{4}$ R. Breun, M. Harper, and B. Cui, Bull. Am. Phys. Soc. 39, 1631 (1994).

${ }^{5}$ D. Ehst and C. F. F. Karney, Nuct. Fusion 31, 1933 (1991).

${ }^{6}$ L. Spitzer and R. Härm, Phys. Rev. 89, 977 (1953).

${ }^{7}$ T. Hellsten and E. Tennfors, Phys. Scr. 30, 341 (1984).

${ }^{8}$ M. Vukovic, Ph.D. thesis, University of Wisconsin, 1995.

${ }^{9}$ L. Villard, K. Appert, R. Gruber, and J. Vaclavik. Comput. Phys. Rep. 4, 95 (1986) 